\title{
Philosophiques
}

\section{Le parvis du temple}

\section{Patrick Drevet}

Volume 22, numéro 1, printemps 1995

URI : https://id.erudit.org/iderudit/027311ar

DOI : https://doi.org/10.7202/027311ar

Aller au sommaire du numéro

Éditeur(s)

Société de philosophie du Québec

ISSN

0316-2923 (imprimé)

1492-1391 (numérique)

Découvrir la revue

Citer cet article

Drevet, P. (1995). Le parvis du temple. Philosophiques, 22(1), 95-102.

https://doi.org/10.7202/027311ar

Ce document est protégé par la loi sur le droit d'auteur. L'utilisation des services d'Érudit (y compris la reproduction) est assujettie à sa politique d'utilisation que vous pouvez consulter en ligne.

https://apropos.erudit.org/fr/usagers/politique-dutilisation/
Cet article est diffusé et préservé par Érudit.

Érudit est un consortium interuniversitaire sans but lucratif composé de l'Université de Montréal, l'Université Laval et l'Université du Québec à Montréal. Il a pour mission la promotion et la valorisation de la recherche. https://www.erudit.org/fr/ 


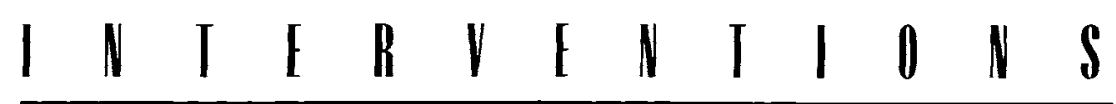

\section{IE PARVIS DU TEMPIE}

\author{
par \\ Patrick Drevet
}

Chacun porte en soi un artiste qui sommeille, plus ou moins. La curiosité qui pousse à s'ouvrir à l'une ou à l'autre des manifestations diverses de l'art est indissociable d'une pointe d'envie ou de regret, voire de jalousie. Une conviction intime assure que, dans d'autres conditions ou à la faveur d'autres conjonctures, on aurait pu cultiver cette fibre originale qui portait d'indistinctes promesses et que l'on sent toujours vibrer, encore qu'elle ait été négligée sous l'urgence d'autres tâches. On ne fréquente pas l'art sans porter au fond de soi une œuvre irréalisée dont le possible, quand on l'imagine, concurrence toutes celles qui le sont.

Cet état contradictoire qui, vis-à-vis de l'art, en rend d'autant plus avide que l'on s'en éprouve plus frustre, s'exacerbe aujourd'hui du fait de l'accessibilité extrême donnée aux ouvres par l'expansion proliférante des moyens de diffusion, lesquels tendent à suggérer leur confection à portée des capacités de tous. Il s'exacerbe aussi du fait de l'impossibilité où l'on se sent d'user de ces mêmes capacités parce que la haute technicité des postes dont on a suivi la filière a conduit à ce qu'on les y aliène.

Tout entier mobilisé dans un but qui n'est pas, intrinsèquement, l'expression de soi, sans doute prend-on conscience d'être ni plus ni moins qu'un esclave contraint à donner le meilleur de lui-mème en vue d'un objectif qui lui apporte certes, en retour, les moyens d'assurer sa subsistance et son confort, mais qui, éloigne et échappant dans sa dimension d'enjeu collectif, l'a détourné de lui, a différé la réalisation d'un désir plus constitutif, a épuisé des facultés et des qualités qu'il n'est plus possible d'exploiter en vue d'une 
valorisation d'un autre ordre que celui de la réussite professionnelle. L'art se présente a contrario comme le mode d'expression rêvé ou tout ce que l'on investit ne sert que l'accomplissement de soi. L'attrait exercé par toute discipline artistique relève d'un désir qui, frustré de la satisfaction donnée tant soit peu par une œuvre effective, s'assouvit par compensation dans la fréquentation des œuvres des autres.

\section{II}

Le succès des rétrospectives, l'affluence aux concerts, l'engouement pour les « salons du livre », la fréquentation croissante des musées, des bibliothèques, des festivals, tout cela ne va pas sans ambiguité dans la mesure où, parallèlement, galeries et librairies sont désertes, salles de cinéma et de théâtre sont boudées, lieux de création et programmes à ambition culturelle perdurent seulement à coups de subventions. L'art semble n'être attractif qu'à proportion de la dimension spectaculaire de ses parades et, alors, lui-même sans doute est-il moins l'objet de la curiosité du public que le public lui-même tant, dans notre civilisation réputée individualiste et anticonformiste, la crainte de s'exclure en ne suivant pas les autres assujettit tout le monde.

Il conviendrait d'analyser ce phénomène et de ne pas s'en tenir à sa constatation, dont il est de bon ton de s'indigner. Il ne pourrait se produire si ne le déclenchait un ressort profond. Le devoir que l'idéologie ambiante fait à tout citoyen de se cultiver ne deviendrait pas une sorte de chantage, avec menaces d'être dèchu de son humanité et promis aux marécages de l'obscurantisme, voire de la bestialité, resterait lettre morte, comme nombre de préceptes opiniâtrement ressassés par les encycliques pontificales sans qu'on voie aucun intégriste s'y conformer davantage sinon en apparence, s'il ne ranimait quelque résolution ancienne, n'éveillait quelque terreur authentique, ne suscitait une amorce de honte et de remords.

Les conversions obtenues ne sont cependant guère plus profondes que la foi du charbonnier. Nos jubilés culturels, qui attirent les foules autant que la communion pascale parce que l'on s'y dédouane de ses manquements de toute l'année, contribuent à donner à l'art une dimension exhibitionniste étrangère à sa nature. Ainsi en vient-on à montrer des tableaux qu'il devient impossible de voir à cause de l'affluence; à exposer des écrivains sinon comme des monstres du moins comme des êtres à part du fait qu'ils sont parvenus à exprimer la bizarrerie que constitue une solitude, sombres hiboux mettant en valeur les spécimens plus rares illuminés du nimbe que leur octroient les faveurs du tube cathodique; à attribuer à des individus élus sur des vertus certainement moins capricieuses que celle du Saint-Esprit le pouvoir de désigner ce qui est le meilleur; et à remplacer la commémoration des saints par celle 
des muses en multipliant les journées, les semaines et les mois consacrés à telle ou telle discipline artistique.

Il n'y a là rien de gratuit ni d'innocent. L'empereur Auguste, qui excellait en matière de dictature, avait déjà par sa formule « Panem et circenses » indiqué le secret de la paix sociale, et nos démocrates ne laissent pas de s'y conformer s'ils ne la répètent pas. Les intentions politiques et économiques qui soustendent les manifestations culturelles vont hélas dans un sens opposé à celui de la culture, mais tout le monde y trouve son compte, à commencer par le public, conforté dans sa propension à l'idolâtrie qui est, somme toute, un confort et une excuse à la paresse.

Nous aimons à penser, en effet que nous n'atteindrons jamais ce que nous croyons la supériorité des èlites. Nous nous bornons à effleurer le champ de leur regard : le fait d'en être remarqué suffit à inoculer une molécule de leur gloire. Le sentiment de notre médiocrité nous incite à vouloir que d'autres, grâce à leurs mérites propres ou par les caprices du sort, mènent une existence à la hauteur des ambitions que nous n'osons pas avoir pour nous-mêmes. Avec les artistes comme avec les stars du cinéma, les rois ou les reines autoproclamés de la chanson, les champions sportifs, les leaders politiques, les héros de la finance, nous vivons par procuration les aventures et les triomphes dont nous ne nous sentons capables qu'en puissance. Nous leur confions la mission de tenir notre place sur la scène de l'Histoire, de nous défendre contre les forces d'anéantissement, de nous donner, bien sûr, l'image la plus avantageuse qui soit de notre espèce. Nous leur demandons d'incarner le rêve que nous n'avons pu réaliser faute d'avidité, de force, de chance, de ruse, de relations, de pouvoir. Nous les situons dans un monde que nous souhaitons mythique, ou à tout le moins privilégie. Nous les voulons hors de la proximité qui nous les montrerait ordinaires. Dès lors, ils peuplent d'étoiles le ciel de nos cerveaux, mais ce n'est plus que cette célébrité qui attire, comme le sont les moucherons par la lumière encore qu'en l'occurrence ce soit une lumière que nous avons nous-mêmes allumée.

L'idolâtrie maintient dans une timidité qui interdit l'approche de l'art. Les artistes vrais seraient les premiers à conseiller autant de candeur et de simplicite devant leurs ouvrages qu'ils en ont eux-mêmes face au monde. Il y a besoin d'une démythification de la culture pour l'aborder mais l'on a aujourd'hui plus de curiosité pour les artistes que pour les œuvres parce que l'on éprouve plus, sans doute, la vocation à l'art, et surtout à la légende qui s'y rattache, que le souci de culture, autrement dit : on aspire moins à se cultiver qu'on ne rêve de devenir « artiste ». 


\section{III}

Dans un monde que ne structure plus l'imaginaire religieux et qui réduit les tâches à ce qu'elles sont, les gestes à des gestes, les aspirations à des aspirations, la matière à la matière et le vide au vide, le désir de transcendance n'en subsiste pas moins, qui oriente vers les productions qu'anime un élan inhérent à la sensibilité. L'idolâtrie fait le succès des manifestations culturelles mais témoigne aussi d'une insatisfaction foncière qui démontre que l'homme, selon le mot de Hölderlin, « est poète ou n'est pas ».

C'est peu dire que nous ne nous contentons pas d'une existence seulement matérielle ou sociale. Longtemps la littérature a été faite par des rentiers dont on pourrait s'étonner qu'ils aient préféré la gestion de leurs chimères à celle de leur fortune. La reconnaissance sociale que leur octroie leur savoir n'exempte pas non plus les professeurs d'un désir de reconnaissance plus universelle. De même, la réussite professionnelle ne suffira jamais à procurer un sentiment d'accomplissement, et peu de ceux qui se sont hissés jusqu'au vedettariat échappent à la tentation d'étendre leur renom au-delà de leur spécialité. Quant aux hommes politiques, même parvenus au faîte du pouvoir...

Nous vivons en permanence sur un plan qui ne coïncide jamais avec celui des activités que nous sommes conduits à mener. Les œuvres d'art font miroiter cet autre territoire comme les réclames des agences de voyage pour des plages lointaines, virginales, paradisiaques. Il n'est pas question seulement de mélancolie. L'art attire comme un aliment qui contiendrait un élément indispensable à notre équilibre et à notre croissance. De façon plus précise, il nourrit en nous une part fondamentale, constitutive de notre être, à l'intersection du langage et du corps. Cette part n'appartient pas toute au langage : c'est précisément parce que l'organisation sociale n'est que langage qu'elle nous laisse frustrés; elle n'appartient pas purement au corps : elle n'est point repue par les satisfactions animales. Elle exige une rencontre de notre raison avec la matière comme c'est le cas précisément, dans l'expression artistique, parce que les idées ni les intentions n'y sont primordiales mais que des aspirations profondes y travaillent et y façonnent une matière.

Quelque perverti que soit l'aspect qu'il prenne, l'attrait pour l'art exprime un besoin irrépressible de proximité avec le monde, le tout du monde, par le moyen des sens, de tous les sens, ce qui inclut l'érotisme dont l'absence prive au contraire d'un ressort essentiel le désir qu'on peut avoir aussi pour les études ou les tâches de la vie active.

\section{IV}

Nous en venons à l'art un peu comme nous entrons dans l'avenir si nous en croyons Paul Valéry : à reculons. Nombre de cinéphiles ont contracté leur 
passion lors de houleuses séances de Ciné-club au cours desquelles leur étaient passés des chefs-d'œuvre qu'ils trouvaient détestables. Beaucoup sont aujourd'hui des fervents de la peinture moderne qui tenaient l'œuvre de Picasso pour de la supercherie. Á quels fidèles abonnés des saisons lyriques eut-il été possible de supporter le moindre air d'opéra dans leur enfance ? Et nous avons tous gardé de nos années scolaires le souvenir de textes soporifiques ou hermétiques dont des fragments reviennent nous hanter, lumineux tout à coup, ou illuminés de l'intelligence acquise par nos facultés et nos sens.

Qui pourrait prétendre avoir profité de tout ce que lui offraient les études? Il est dans l'ordre des choses que nous restions vis-à-vis de l'art des amateurs. Plus nous avançons en âge, plus nous mesurons le temps que nous avons perdu, plus nous nous résignons aussi à ne jamais disposer du savoir, des méthodes et des outils nécessaires à l'appréhension de ce qui, avec le recul. nous apparaît essentiel et saurait maintenant donner plus de poids à notre pensée, plus d'acuité à notre perception, plus de sagacité à notre vision, plus d'économie à notre comportement, plus d'intensité à nos relations, plus de vie à notre vie, plus d'ètre à notre être.

La timidité avec laquelle nous approchons les ouvrages qui relèvent de l'art, quand elle ne nous en interdit pas carrément l'accès, vient du sentiment de ce retard, et la conviction qu'il est inexorable renforce la paresse que nous avons à reprendre un apprentissage qui demeure toujours possible. Sans doute est-ce aussi une façon d'expliquer que nous ne soyons pas devenus plus que ce que nous nous trouvons être, d'avouer que nous avons été infidèles au possible que nous portions en nous et qui reste, intrinsèquement, plus que ce que nous sommes. C'est conférer à l'art, à tort ou à raison, le statut d'archétype de l'accomplissement, avec tous les effets pervers que nous voyons en résulter dans la dimension idolâtre que peut revêtir la dévotion aux « génies ».

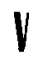

Il est clair que c'est en prenant conscience de sa bêtise que l'on devient intelligent. Recenser ses manques suppose une maîtrise que l'on approcherait vraiment si l'on avait tout appris, mais reconnaître ses limites prouve déjà une ouverture d'esprit qui rend disponible à tout. Depuis Socrate au moins, les savants et les sages n' ont cessé de le répéter au terme de leurs découvertes. Il ne faut donc pas s'étonner que la curiosité pour l'art donne l'impression de venir toujours un peu tard, et trop tard. L'école n'était pas le lieu pour la donner, ni même les études supérieures sans doute. Il suffit que celles-ci structurent les facultés, développent les capacités, inculquent des savoir-faire. Leur mission n'est atteinte, précisément, qu'à proportion de l'insatisfaction où elles laissent, de l'état d'avidité auquel elles conduisent, du désir qu'elles ont su agrandir. Les diplômes qu'elles distribuent enregistrent ou devraient 
enregistrer seulement l'excellence du fonctionnement : ils signifient qu'une autonomie a été acquise, d'ordre technique plus qu'existentiel. L'épanouissement de notre « potentiel cognitif » ne nous donne rien à connaître ni à comprendre, c'est-à-dire rien que nous puissions prendre avec nous et au contact de quoi nous naissions enfin. Les études, mais aussi bien tout apprentissage, et les mœurs, et l'expérience, façonnent des vases, ils restent à remplir.

Éprouver de l'intérêt pour la culture suppose, en somme, que l'on soit déjà « cultivé », mais chercher à se cultiver davantage comme on l'a fait par ses études ne saurait aboutir qu'à perfectionner une machine qui tournerait à vide. En nous-mêmes nous ne pouvons exercer et rendre performantes que nos facultés, nos capacités, l'acuité de nos sens. Il est une pratique de la culture qui s'apparente à celle du culturisme, lequel consiste à développer les muscles sans autre but que leur développement, avec pour résultat de ne proposer que de répétitives planches d'anatomie. La culture ne saurait être elle-même son propre sens. Ce n'est pas amoindrir ses manques et reculer ses limites qu'accumuler indefiniment des savoir-faire.

Si l'attrait pour l'art témoigne de l'œuvre en germe ou en latence que nous portons en nous, il confirme aussi bien une instruction achevée, une éducation aboutie. Il signifie que l'intégralité de la personne « fonctionne », avec l'entière liberté de comportement et de choix qui lui échoit. Ce qui n'est pas l'inconnu pour elle alors le devient, ou du moins prend un relief particulier, son existence l'alerte, ses formes suscitent l'envie d'une fréquentation plus éclairée, appelle à une connaissance approfondie.

On se tourne vers l'art parce qu'on se suffit tant soit peu à soi-même ou parce qu'on se sent tout au moins suffisamment suffisant pour n'avoir plus à entretenir le souci de soi et pour ressentir le besoin d'aller voir ailleurs. On naît à la curiosité quand il devient vain de ne penser qu'à soi. On prend goût à l'altérité quand les facultés épanouies disposent naturellement à l'accueil. Et l'on se tourne vers l'art parce qu'en son essence l'art est l'altérité. Ce n'est surtout pas soi qu'il y faut aller chercher. Ce qu'il nous dit sur nous-mêmes relève des mediations qu'il emploie pour se traduire. C'est probablement ce que Baudelaire exprimait dans sa définition : « Le Beau est le Bizarre ». Il y a une irréductibilité en chaque œuvre qui en constitue à la fois la valeur insigne et la séduction inouie.

L'attrait de l'art pourrait se résumer au désir d'accéder à ce qui subjugue. Ou, selon une formule heureuse d'Edgar Morin à propos du discours amoureux : « posséder ce qui nous possède ». Nous pouvons d'abord, plus profondément, aspirer au désir d'être subjugué, c'est-à-dire souhaiter en nous une ouverture qui nous sorte de nous, revendiquer le besoin de ce dont nous n'avons pas besoin. 


\section{VI}

Le parvis du Temple est encombré de génies qui ne sont point morts encore que leur socle est serré déjà dans la forêt de statues qui distraient d'y entrer. Parmi des sermonneurs qui brandissent leurs volumes, certains par leur faconde, leur habileté, leur charme physique se partagent la foule qui pour sa plus grosse part s'en tient alors à eux. Il ne manque pas non plus de vendeurs d'idées reçues exposant miroirs aux alouettes, poudre aux yeux, copies, pièges à gogos, chimères et autres oiseaux de fable. Des fidèles affluent sur des motivations douteuses. L'édifice lui-même, grandiose, brillant, présente plus de force attractive que ce au nom de quoi il a été bâti et qui est insaisissable.

Les productions artistiques encourent le risque d'attirer plus pour la gloire qui les entoure que pour la médiation qu'elles ont été et proposent. Nul n'est à l'abri de verser dans cette attitude qui a ses racines dans les siècles, qui nous a étè transmise par l'éducation, qui s'appuie sur les valeurs de notre civilisation que le perfectionnement des outils médiatiques aujourd'hui exacerbe. Que la somme atteinte par Les toumesols de Van Gogh declenche un intêrêt unanime pour ce peintre, la réalisation de films inspirés de sa vie, l'organisation d'expositions de ses toiles, et des fantasmes de toutes sortes sur sa figure relève d'un frivole effet de mode plus que d'un sincère éveil à la peinture. Or tout artiste authentique n'est jamais qu'un artisan avant d'être le génie que l'on proclame pour des raisons qui ne tiennent pas à l'art mais à la psychologie des foules. Si tant est qu'on veuille assouvir sa soif d'art, un sain réflexe devrait être de ne pas prendre le flacon pour la boisson, autrement dit de faire fi de l'image sacrée et aveuglante qu'eux-mêmes ou leurs zélateurs donnent des «phares », et alors même que, dans un élan tout romantique, certaines de nos gloires contemporaines flattées dans leur vanité sont les premières à se prendre pour ce qu'elles sont et s'isolent dans des attitudes de divinités.

L'attrait pour l'art manifeste chez les créateurs qui s'y adonnent comme chez les amateurs qui le « consomment » une attente qui ne saurait être comblée par aucune autre médiation, et cette attente signale au fond de chacun une vacance dont la nature recouvre l'acception que les théologiens donnent à l'état de pécheur. Pécheur est celui qui ne se suffit pas à lui-même, qui manque d'être pour éprouver, pour comprendre, pour atteindre les objectifs qu'il se fixe. pour être, parce qu'il demeure toujours en-deça, voire à l'opposé, des idées de Beauté, de Bonheur, d'Amitiè, ou d'Amour pourtant inscrites en lui.

Se tourner vers l'art exprime la nécessité que l'on éprouve plus ou moins à son insu de traiter avec sa solitude, avec ses appétences secrètes, si secrètes qu'elles le sont à soi-même. C'est prendre en compte la part obscure, immémoriale, ou irréductible, que nous sommes astreints à laisser de côté dans les relations sociales, cette part inexprimée et inexprimable sur laquelle nous ne pouvons échanger même avec des proches, et à laquelle il nous faut 
néanmoins consacrer du temps pour ne pas traîner avec nous un mécontentement indistinct, un dégoût inexplicable, une frustration handicapante, voire des symptômes de sclérose.

Ce nécessaire retour sur nous porte de façon paradoxale sur ce qui nous est autre. Ce que nous pouvons attendre de l'art et que nous y cherchons n'est pas ce que nous possédons déjà, cela ne saurait nous manquer. L'agacement ou l'envie dont ne se départ pas la satisfaction esthétique est bien souvent l'indication que nous sommes conquis et que nous aimons. Initiation de la jalousie : je ne posséderai jamais telle faculté, telle capacité, tel talent, pas plus que je ne possède telle figure, tel corps, telle couleur d'yeux ou de chevelure; mais je le possède en définitive plus que l'autre lui-même puisqu'il me l'offre comme jamais il ne pourra se l'offrir à lui-même.

L'art est le mode d'expression auquel tout le monde se sent peu ou prou appelé parce qu'il ne délaisse aucune des fibres qui nous constituent, intellectuelle comme sensuelle, sentimentale comme érotique. Il ne compartimente pas notre personne dont il sollicite au contraire la totalité. Pour autant, il n'est l'expression de rien, si ce n'est celle d'un manque et d'une tension : il est appel à créer, cest-à-dire à imposer au monde des objets, des formes, des sons qui n'existent pas. Ces objets, ces formes, ces sons ne subsisteront pas : le monde de l'art est un champ de ruines. Et l'intérieur du Temple est vide. Il célèbre le désir de créer dont il est le moyen le plus propre ả l'assouvir parce que tout ce que l'on y investit ne sert que ce désir.

\section{Ecrivain}

Paris 